\title{
Repeated Heat Exposure Impairs Nigrostriatal Dopaminergic Neurons in Mice
}

\author{
Hyo Geun Kim, ${ }^{a}$ Tae-mi Kim, ${ }^{a}$ Gunhyuk Park, ${ }^{b}$ Tae Hee Lee, ${ }^{c}$ and Myung Sook Oh*,a,b \\ ${ }^{a}$ Department of Oriental Pharmaceutical Science, College of Pharmacy, Kyung Hee University; ${ }^{b}$ Department of \\ Life and Nanopharmaceutical Sciences and Kyung Hee East-West Pharmaceutical Research Institute, Kyung Hee \\ University; \#1 Hoegi-dong, Dongdaemun-gu, Seoul 130-701, Republic of Korea: and ${ }^{c}$ Department of Formulae \\ Pharmacology, School of Oriental Medicine, Gachon University; \#1342 Seongnamdaero, Sujeong-gu, Seongnam \\ 461-701, Republic of Korea. \\ Received April 6, 2013; accepted June 28, 2013; advance publication released online July 10, 2013
}

Environmental heat stress is associated with physical stress responses, including changes in monoamines, protein expression, and neuronal circuits and damage to neurons in the brain. This study determined the effects of heat stress on the nigrostriatal dopaminergic system based on behavioral, histological, and neurochemical analyses. To evaluate behavioral changes after heat exposure, we subjected mice to the pole and open field tests. The data suggested that heat stress for $7 \mathrm{~d}$ significantly impaired movement. Then, we conducted a histological analysis using tyrosine hydroxylase (TH) immunoreactivity in the striatum and substantia nigra (SN). Heat stress induced a significant deficit in TH-positive fibers and cells after 14- and 21-d exposure, respectively. We also measured the striatal dopamine (DA), 4-hydroxy-3-methoxy-phenylacetic acid, and 3,4-dihydroxyphenylacetic acid levels. The data suggested that DA turnover rate increased with heat exposure in a time-dependent manner, resulting in the significant decrease of DA after $28 \mathrm{~d}$. Moreover, the expression of heat shock protein 70 (HSP70) was increased in the mouse SN with up to 14-d heat exposure, but decreased after $21 \mathrm{~d}$ of the stress. And glucose-regulated protein 78 (GRP78) was gradually increased in the mouse SN with 28-d heat exposure. The caspase-3 activity was also increased after 14-d heat exposure. These findings are the first evidence that repeated heat stress impairs nigrostriatal dopaminergic neurons, motor function, and DA availability with changes of HSP70 and GRP78 expressions and caspase-3 activity in mice.

Key words repeated heat stress; dopaminergic neuron; heat shock protein 70; glucose-regulated protein 78; caspase-3

Extreme environmental temperatures induce systemic metabolic disorders accompanied by hyperthermia, behavioral, physiological, and biochemical changes, which might be considered pathophysiological indices for assessing heat stress. ${ }^{1,2}$ For example, heat stress induced changes in the blood glucocorticoid levels, vasopressin release, heat adaptation, as well as increases in blood pressure and heart rate, hypoglycemia, gastric hemorrhage, and spermatogenic dysfunction. ${ }^{3,4)}$ Studies have demonstrated that the brain is very vulnerable to heat; hyperthermia can lead to monoamine overload, neural circuit modification, neuron loss, neurological defects, convulsions, and heat stroke. ${ }^{5-9)}$ Additionally, hyperthermia can aggravate brain damage induced by stroke, traumatic brain injury, and certain drugs. ${ }^{10)}$

Dopaminergic neurons are the main source of dopamine, which is one of the most intensively studied neurotransmitters in the brain due to its involvement in several neurological and psychiatric disorders such as Parkinson's disease (PD), schizophrenia, and attention deficit hyperactivity disorder. ${ }^{11,12)}$ Among the several dopaminergic pathway, the nigrostriatal system in the midbrain is the best-known. It originates in the zona compacta of the substantia nigra (SN) and extends its fibers into the dorsal striatum, and it plays an essential role in the control of voluntary motor movement. ${ }^{11)}$ For example, the dorsal striatum receiving input from the motor cortex participates in the skeletomotor loop that crucially controls voluntary movements. ${ }^{13)}$ Numerous studies have indicated an association between dopaminergic system dysfunction and

The authors declare no conflict of interest. stress such as that caused by not only neurotoxins but also environmental toxins. ${ }^{12)}$ For example, heat exposure at $34^{\circ} \mathrm{C}$ for $14 \mathrm{~d}$ reduced the content of tyrosine hydroxylase $(\mathrm{TH})$, a rate-limiting enzyme in the biosynthesis of dopamine, of the $\mathrm{SN}$ in rats $^{5)}$ and heat exposure at $43^{\circ} \mathrm{C}$ for $2 \mathrm{~h}$ induced striatal neuron dysfunction gradually in vitro. ${ }^{7,10}$ From the previous results, we assumed that hyperthermia could damage striatal dopaminergic neurons in mice and few studies have examined the functional changes in the striatal dopaminergic system after heat exposure in vivo.

Therefore, this study compared the time-dependent effects of heat stress on motor function, dopaminergic neuron survival, and the levels of dopamine and its metabolites especially in nigrostriatal pathway in mice. Additionally, to understand the neurotoxicity of heat stress, changes in the expressions of heat shock protein 70 (HSP70) and glucose-regulated protein 78 (GRP78) and in the activation of caspase-3 were measured.

\section{MATERIALS AND METHODS}

Materials Tetramethylethylenediamine, the protein assay, Tween 20, ammonium persulfate, acrylamide, enzyme-linked chemiluminescence reagent (ECL) reagent, and skim milk were purchased from Bio-Rad (Hercules, CA, U.S.A.). Rabbit anti-TH was obtained from Millipore (Billerica, MA, U.S.A.). Rabbit anti-GRP78 was obtained from Abcam (Cambridge, MA, U.K.). Caspase-3 assay kit was purchased from BioVision (Mountain View, CA, U.S.A.). Anti-rabbit-horseradish peroxidase (HRP) secondary antibody was purchased from Assay Designs (Ann Arbor, MI, U.S.A.). Biotinylated anti-rabbit 
antibody, normal goat serum, and the avidin-biotin peroxidase complex $(\mathrm{ABC})$ standard kit were purchased from Vector Laboratories (Burlingame, CA, U.S.A.). All other reagents, including dopamine (DA) hydrochloride, 4-hydroxy-3-methoxyphenylacetic acid (HVA), and 3,4-dihydroxyphenylacetic acid (DOPAC) were purchase from Sigma-Aldrich (St. Louis, MO, U.S.A.).

Animals and Heat Exposure Male ICR mice (7 weeks, 30-32 g) were purchased from Daehan Biolink (Eumseong, Korea). The mice were divided randomly into seven groups of 11 or 12: the (1) normal (normothermia) and the (2) 1-d, (3) 4-d, (4) 7-d, (5) 14-d, (6) 21-d, and (7) 28-d groups. Groups (2) to (7) were exposed to heat stress once a day for $1,4,7$, 14,21 , or $28 \mathrm{~d}$, respectively. The animals were housed four per cage, had free access to water and food, and were kept under a constant temperature $\left(23 \pm 1^{\circ} \mathrm{C}\right)$, humidity $(60 \pm 10 \%)$, and a 12-h light/dark cycle. Animal treatment and maintenance were carried out in accordance with the Principles of Laboratory Animal Care (NIH publication No. 85-23, revised 1985) and the Animal Care and Use Guidelines of Kyung Hee University, Seoul, Korea. Within 5 weeks of arrival, the mice were adapted to their surroundings for $7 \mathrm{~d}$ and kept under the same conditions before the study. To avoid the influence of diurnal cycling, heat exposure was started at approximately the same time each day between 9 and $10 \mathrm{a} . \mathrm{m}$. Heat exposure was achieved by transferring the mice in their cage into a chamber (Jeio Tech, Daejeon, Korea) at $43^{\circ} \mathrm{C}$ and $60 \pm 10 \%$ humidity for $15 \mathrm{~min}$ once a day. The heat condition was slightly modified from Yang et al., ${ }^{14)}$ based on survival rate. In preliminary test, mice with up to $15 \mathrm{~min}$ heat exposure at $43^{\circ} \mathrm{C}$ easily recovered in $10 \mathrm{~min}$ without death at room temperature. However, after $18 \mathrm{~min}$ to $25 \mathrm{~min}$ of heat stress at $43^{\circ} \mathrm{C}$, dead mice were found in our ambient chamber. After the stress, the mice were moved back to room temperature. We performed the behavioral tests $12 \mathrm{~h}$ after the last heat exposure.

Pole Test and Open Field Test In the pole test, the mice were placed head upward on near the top of a rough surface pole (a stainless pole with gauze-taped, diameter $8 \mathrm{~mm}$, height $55 \mathrm{~cm}$ ). The times required to turn around completely and then climb down until all four feet reached the floor were recorded. Each trial had a cut-off limit of $60 \mathrm{~s}$. For the locomotor activity, all measurements were made between 9 p.m. and 2 a.m. to avoid diurnal variation. The mice were placed in the testing chamber $(40 \times 25 \times 18)$ with black floors for $15 \mathrm{~min}$ adaptation, followed by a 60-min recording period using a computerized automatic analysis system (Viewer; Biobserve, Bonn, Germany). The data collected by computer included the total distance traveled by tracking the center of the animal.

Brain Tissue Preparation At next day after last heat exposure, mice were perfused transcardially with $0.05 \mathrm{M}$ phosphate buffered saline (PBS), and then fixed with cold $4 \%$ paraformaldehyde (PFA) in $0.1 \mathrm{~m}$ phosphate buffer. Brains were removed and post-fixed in $0.1 \mathrm{M}$ phosphate buffer (PB) containing $4 \%$ paraformaldehyde overnight at $4{ }^{\circ} \mathrm{C}$ and then immersed in a solution containing $30 \%$ sucrose in $0.05 \mathrm{M}$ PBS for cryoprotection. Serial $30 \mu \mathrm{m}$-thick coronal sections were cut on a freezing microtome (Leica, Nussloch, Germany) and stored in cryoprotectant $(25 \%$ ethylene glycol, $25 \%$ glycerol, and $0.05 \mathrm{M} \mathrm{PB}$ ) at $4{ }^{\circ} \mathrm{C}$ until use. For high performance liquid chromatography (HPLC) and Western blot analysis, the mice were decapitated and the brains were isolated and stored at
$-80 \circ \mathrm{C}$ until use.

Measurement of Neurotransmitter Levels by HPLC The tissue contents of the neurotransmitter were measured using HPLC (Dionex Corp., Synnyvale, CA, U.S.A.) in combination with an electrochemical detecting system (ESA Coulochem III detection system, range $100 \mathrm{nA}$, potential $-50 \mathrm{mV}$ to $+300 \mathrm{mV}$; ESA Inc., Chelmsford, MA, U.S.A.). The striatum tissue was homogenized in $0.2 \mathrm{M}$ perchloric acid. Homogenates were centrifuged for $20 \mathrm{~min}$ at $0^{\circ} \mathrm{C}$ and $14000 \times \boldsymbol{g}$. The supernatant was filtered through a $0.22 \mu \mathrm{m}$ membrane, and an aliquot ( $10 \mu \mathrm{L}$ in volume) of the resulting solution was injected into the HPLC pump. Chromatographic separation was performed using a C18 reverse-phase column $(150 \times 3 \mathrm{~mm}, 3 \mu \mathrm{m}$; Thermo Scientific, San Jose, CA, U.S.A.) at $25^{\circ} \mathrm{C}$, and data analyses were performed using Chromeleon ${ }^{\mathrm{TM}}$ software (Version 6.40) from Dionex Corp. The mobile phase, which had a $\mathrm{pH}$ of 4.0 , consisted of $150 \mathrm{~mm}$ ammonium acetate, $140 \mu \mathrm{M}$ ethylenediaminetetraacetic acid, 15\% methanol, and 5\% acetonitrile. The flow rate was maintained at $0.2 \mathrm{~mL} / \mathrm{min}$ and the temperature of the column was $25^{\circ} \mathrm{C}$. DA, DOPAC, and HVA standards were prepared in $0.2 \mathrm{M}$ perchloric acid and each concentration was adjusted with respect to the standard and quantified from a standard curve. The levels of DA, DOPAC, and HVA were calculated as nanograms per microgram of total protein which was determined with the protein assay.

Immunohistochemistry and Quantification For immuonhistochemical study, brain sections were briefly rinsed in PBS and treated with $1 \%$ hydrogen peroxide for $15 \mathrm{~min}$. The sections were incubated with a rabbit anti-TH antibody ( $1: 2000$ dilution) overnight at $4^{\circ} \mathrm{C}$ in the presence of $0.3 \%$ triton X-100 and normal goat serum. After rinsing in PBS buffer, the sections were then incubated with biotinylated antirabbit immunoglobulin $\mathrm{G}$ ( $\mathrm{IgG})(1: 200$ dilution) for $90 \mathrm{~min}$ and with $\mathrm{ABC}(1: 100$ dilution) for $1 \mathrm{~h}$ at room temperature. Peroxidase activity was visualized by incubating sections with 3,3-diaminobenzidine in $0.05 \mathrm{M}$ Tris-buffered saline $(\mathrm{pH}$ 7.6). After several rinses with PBS, sections were mounted on gelatin-coated slices, dehydrated, and cover-slipped using histomount medium. The optical density of TH-immunoreactivty in the striatum was analyzed with ImageJ software (Bethesda, MD, U.S.A.). For measurement of the optical density, the total region of interest was manually outlined and averaged optical densities were acquired in images with converted 8-bit indexed color. The number of TH-immunoreactive (IR) cell bodies in the $\mathrm{SN}$ was counted. The images were photographed at $40 \times$ or $100 \times$ magnification using an optical light microscope (Olympus Microscope System BX51; Olympus, Tokyo, Japan) equipped with a $20 x$ objective lens. Data are presented as percentages of sham group values.

Western Blot Analysis The SN tissues were lysed using a protein assay according to the manufacturer's instructions. The lysates were separated by $10 \%$ sodium dodecyl sulfatepolyacrylamide gel electrophoresis, and then transferred to a membrane. The membranes were incubated with $5 \%$ skim milk in Tris-buffered saline with tween 20 for $1 \mathrm{~h}$ and then with the primary antibody $(1: 1000$ dilution of HSP70) overnight at $4^{\circ} \mathrm{C}$; this was followed by incubation with HRPconjugated secondary antibody for $1 \mathrm{~h}$. Immunoreactive-bands were detected using an ECL detection kit and an LAS-4000 mini system (FUJIFILM, Tokyo, Japan) was used for visualization. The intensities of the bands were normalized to the 

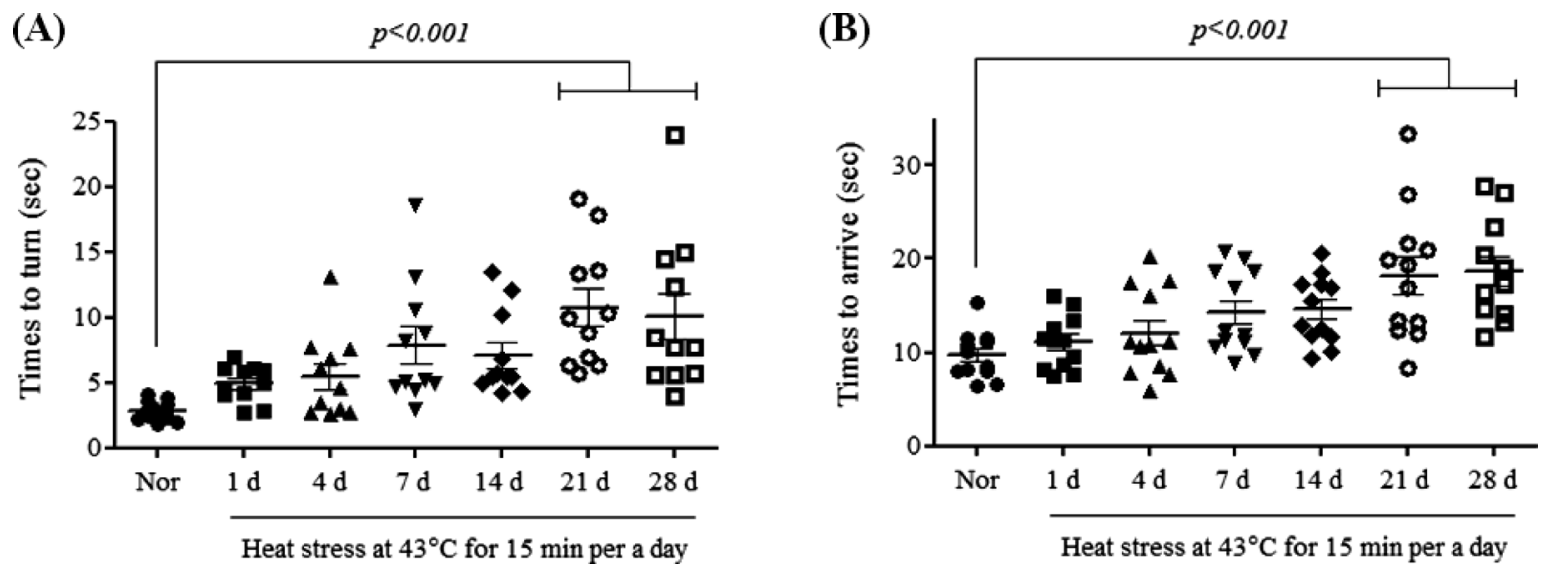

Fig. 1. Effect of Heat Stress on Behavioral Impairment Using a Pole Test

After the mice were exposed to heat for different numbers of days, we conducted the pole test. The times to turn completely downward on the pole and to arrive on the floor (A and B, respectively) were recorded $(n=11-12)$. Values are given as the mean \pm S.E.M. and compared with the normal group.

$\beta$-actin intensity using Multi Gauge software (FUJIFILM).

Measurement of Caspase-3 Activation The caspase-3 activity assay was performed according to the manufacturer's protocol. Briefly, lysates of the SN were incubated with reaction buffer containing $10 \mathrm{~mm}$ dithiothreitol and $1 \mathrm{~mm}$ DEVDAFC substrate. The mixture was incubated for $2 \mathrm{~h}$ at $37{ }^{\circ} \mathrm{C}$ before protease activity was detected using a fluorescence microplate reader, with $360 \mathrm{~nm}$ excitation and $450 \mathrm{~nm}$ emission filters.

Statistical Analysis All statistical parameters were calculated using Graphpad Prism 5.0 software (San Diego, CA, U.S.A.). Values were expressed as the mean \pm standard error of the mean (S.E.M.). Results were analyzed by one-way analysis of variance followed by the Tukey's post hoc test. Differences with a $p$-value less than 0.05 were considered statistically significant.

\section{RESULTS}

Repeated Heat Stress Impaired Motor Function and Striatal Dopaminergic Neurons To investigate the effect of heat stress on behavior, we performed the pole test and open field test. In the pole test, the 21- and 28-d heat-exposure groups showed bradykinesia, taking $10.8 \pm 1.4$ and $10.0 \pm 1.8 \mathrm{~s}$, respectively, to turn on the top of the pole (Fig. 1A; $F(6$, $70)=5.996, p<0.001)$ and $18.2 \pm 2.1$ and $18.6 \pm 1.7 \mathrm{~s}$ to descend (Fig. 1B; $F(6,76)=6.499, p<0.001$ ). There were no significant differences in the time needed to turn and descend between the normal group and the 1- to 14-d heat-exposure groups. Exposure to heat for more than $7 \mathrm{~d}$ also significantly reduced the movement distance in the open field test, with distances of $4773.3 \pm 451.9$ to $4527.4 \pm 322.5 \mathrm{~cm}$ in the heat-exposure groups versus $6513.0 \pm 220.6 \mathrm{~cm}$ for the normal group (Fig. 2; $F(6,42)=5.267, p<0.001)$. Moreover, a significant depletion of the numbers of TH-positive cells and fibers in the SN and striatum, respectively, was observed after repeated heat stress for $21 \mathrm{~d}$ (Fig. 3A; $F(6,28)=35.34, p<0.001, \mathrm{~B} ; F(6,28)=7.227$, $p<0.001$, and $\mathrm{C})$. The 21- and 28-d heat-exposure groups had significantly fewer $\mathrm{TH}$-positive neurons in the SN $(73.8 \pm 7.3$ and $73.3 \pm 4.6 \%$ of the normal group, respectively) compared with controls. In the striatum, the 21- and 28-d heat-exposure

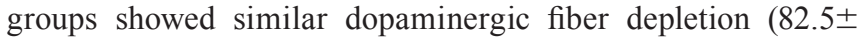

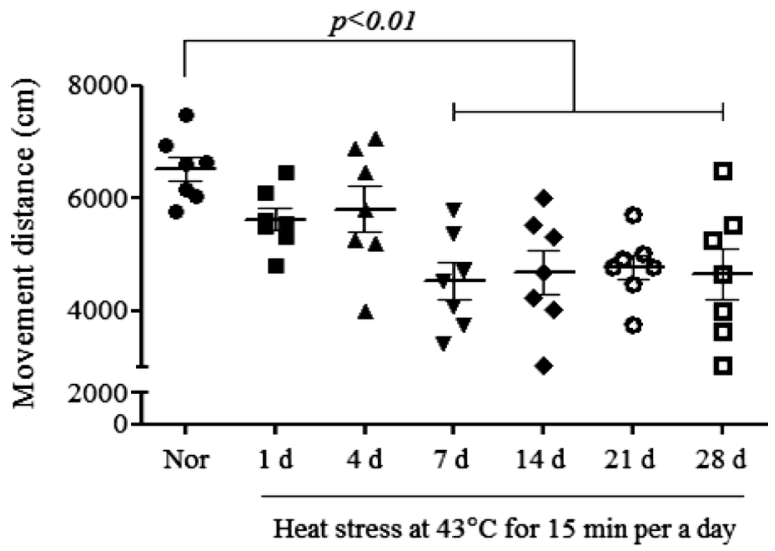

Fig. 2. Effect of Heat Stress on Behavioral Impairment Using an Open Field Test

After the mice were exposed to heat for different numbers of days, we conducted the open field tests. The movement distance in the test chamber was measured $(n=7)$. Values are given as the mean \pm S.E.M. and compared with the normal group.

9.0 and $53.7 \pm 15.2 \%$ of the normal group, respectively). These results demonstrate that repeated exposure to heat significantly induced motor dysfunction and dopaminergic neuron degeneration.

Repeated Heat Stress Decreased Dopamine Availability in the Striatum To examine the effect of heat stress on the dopaminergic system, the levels of striatal DA and its metabolites DOPAC and HVA were measured using HPLC with electrochemical detection (Table 1). This revealed that heat stress induced changes in striatal DA levels after the 4-d exposure $(169.0 \pm 4.3,158.2 \pm 4.3,143.8 \pm 4.0,116.1 \pm 5.5$, and $89.8 \pm 2.4 \mathrm{ng} /$ $\mathrm{mg}$ protein in the 4-, 7-, 14-, 21-, and 28-d groups, respectively) compared with the normal group $(133.3 \pm 9.8 \mathrm{ng} / \mathrm{mg}$ protein; $F(6,28)=18.41, p<0.001)$. There were significant differences in striatal DOPAC levels between the normal group (20.8土 $0.9 \mathrm{ng} / \mathrm{mg}$ protein) and 4-, 7-, and 14-d heat-exposure group $(28.5 \pm 1.0,27.9 \pm 1.0$, and $31.6 \pm 2.2 \mathrm{ng} / \mathrm{mg}$ protein, respectively; $F(6,28)=14.14, p<0.001)$, and there was a significant increase in HVA in the 14-d heat-exposure group $(7.9 \pm 0.7 \mathrm{ng} / \mathrm{mg}$ protein) compared with the normal group $(5.3 \pm 0.2 \mathrm{ng} / \mathrm{mg}$ protein; $F(6,28)=8.00, p<0.001)$. Additionally, there were significant increases in the dopamine turnover ratios (DOPAC/DA; $F(6$, 
(A)

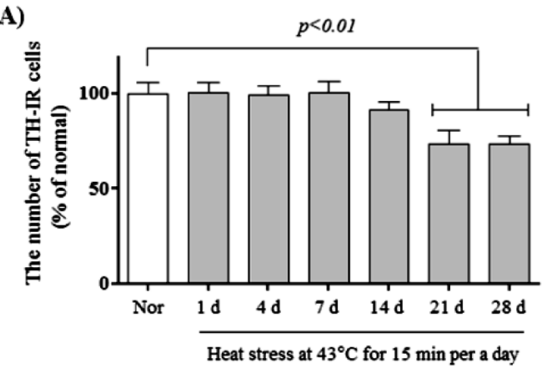

(B)

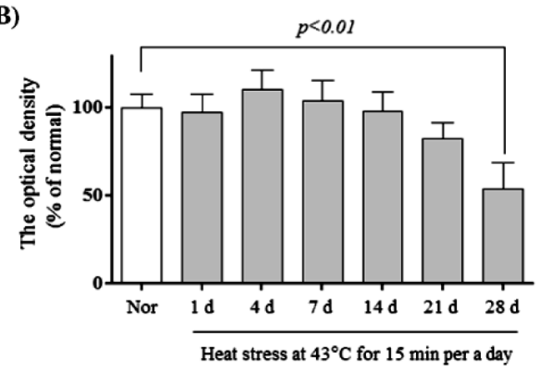

(C)
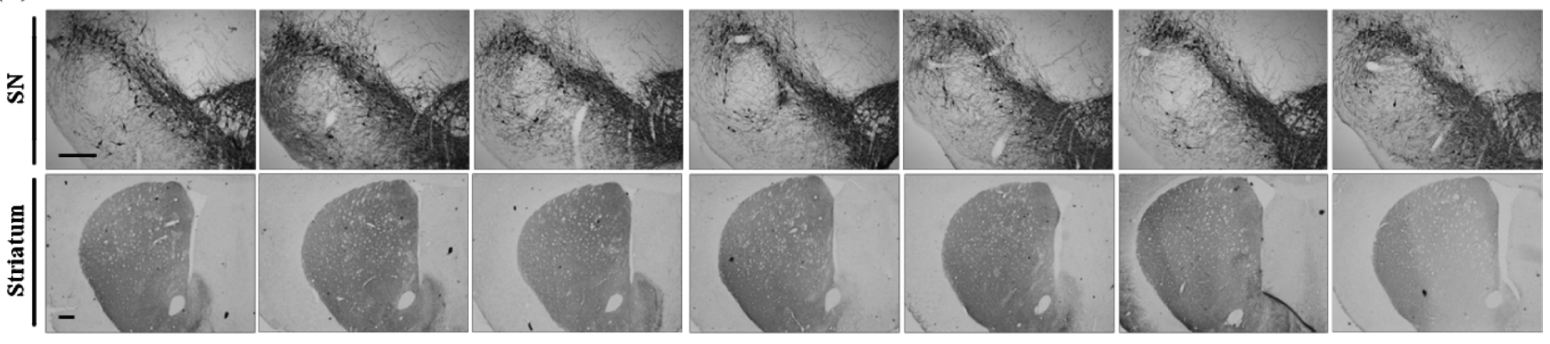

4-day

7-day

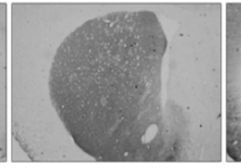

14-day

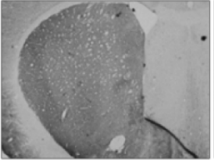

21-day

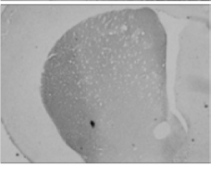

Fig. 3. Effect of Heat Stress on Dopaminergic Neuron Dysfunction in the SN and Striatum

After the mice were exposed to heat for different numbers of days, the mice were perfused and the brain tissues were cryosectioned for immunohistochemistry. The dopaminergic cells were quantified by counting the number of TH-IR cells in the SN (A, $n=5)$ and measuring the optical density in the striatum (B, $n=5)$. Representative photomicrographs are shown (C). Scale bar $=200 \mu \mathrm{m}$. Values are given as the mean \pm S.E.M. and compared with the normal group.

Table 1. Effect of Heat Stress on Changes in Neurotransmitter Levels (ng/mg Protein) in the Striatum

\begin{tabular}{cllllll}
\hline \hline Groups & \multicolumn{1}{c}{ DA } & \multicolumn{1}{c}{ DOPAC } & \multicolumn{1}{c}{ HVA } & DOPAC/DA & HVA/DA & DOPAC+HVA/DA \\
\hline Normal & $133.33 \pm 9.80$ & $20.82 \pm 0.88$ & $5.33 \pm 0.21$ & $0.158 \pm 0.006$ & $0.040 \pm 0.002$ & $0.198 \pm 0.008$ \\
1 -d & $131.66 \pm 9.04$ & $19.00 \pm 1.19$ & $4.89 \pm 0.44$ & $0.145 \pm 0.005$ & $0.037 \pm 0.002$ & $0.182 \pm 0.006$ \\
4-d & $169.01 \pm 4.25^{* *}$ & $28.51 \pm 0.97^{* *}$ & $6.96 \pm 0.33$ & $0.169 \pm 0.006$ & $0.041 \pm 0.002$ & $0.210 \pm 0.007$ \\
7-d & $158.16 \pm 4.25$ & $27.88 \pm 0.97^{* *}$ & $6.62 \pm 0.25$ & $0.176 \pm 0.005$ & $0.042 \pm 0.001$ & $0.218 \pm 0.005$ \\
$14-\mathrm{d}$ & $143.84 \pm 3.99$ & $31.56 \pm 2.18^{* * *}$ & $7.88 \pm 0.70^{* * *}$ & $0.219 \pm 0.012^{* * *}$ & $0.054 \pm 0.003^{* *}$ & $0.273 \pm 0.014^{* * *}$ \\
21-d & $116.08 \pm 5.46$ & $25.19 \pm 1.23$ & $6.88 \pm 0.29$ & $0.218 \pm 0.011^{* * *}$ & $0.060 \pm 0.003^{* * *}$ & $0.278 \pm 0.014^{* * *}$ \\
28-d & $89.77 \pm 2.41^{* *}$ & $21.74 \pm 0.47$ & $5.64 \pm 0.10$ & $0.243 \pm 0.011^{* * *}$ & $0.063 \pm 0.001^{* * *}$ & $0.306 \pm 0.012^{* * *}$ \\
\hline
\end{tabular}

Values are given as the mean \pm S.E.M. $(n=5)$. $* * * p<0.001$ and $* * p<0.01$ compared with the normal group.

$28)=18.95, p<0.001, \mathrm{HVA} / \mathrm{DA} ; F(6,28)=22.88, p<0.001$, and DOPAC+HVA/DA; $F(6,28)=22.17, p<0.001)$ in the 14-, 21-, and 28-d heat-exposure groups in comparison with the normal group. These results indicate that repeated heat stress gradually induced the depletion of total striatal DA but significantly increased dopamine turnover, which might result in impaired motor function.

Repeated Heat Stress Reduced the Expression of HSP70 and GRP78 and Increased Caspase-3 Activation Generally, hyperthermia modulates HSP70, and the fundamental function of the protein is cellular thermotolerance or cytoprotection. $^{8)}$ Therefore, we examined changes in HSP70 expression resulting from repeated heat exposure using western blot analysis. For mice subjected to heat stress for up $14 \mathrm{~d}$, the HSP70 expression in the $\mathrm{SN}$ increased in comparison with the normal group. However, heat stress lasting for 21 and $28 \mathrm{~d}$ reduced HSP70 expression to $101.4 \pm 10.4$ and $81.3 \pm 6.0 \%$ of the normal group, respectively (Fig. 4A; $F(6,28)=91.22$, $p<0.001$ ). Additionally, under a variety of environmental and physiological stress conditions, GRP78 synthesis can be stimulated. ${ }^{15}$ For mice subjected to heat stress, the GRP78 expression in the SN significantly increased to $267.2 \pm 39.9$ and $292.0 \pm 24.4 \%$ of the normal group in the $21-$ and $28-\mathrm{d}$ heat-exposure groups, respectively (Fig. 4A; $F(6,28)=43.76$, $p<0.001)$. Moreover, the activity of a downstream effector of apoptosis, caspase-3, was increased to $164.95 \pm 6.6,171.03 \pm$ 6.6, and 192.2 \pm 3.7 in the 14-, 21-, and 28-d heat-exposure groups, respectively, versus activity of $100.0 \pm 5.95$ in the normal group (Fig. 4C; $F(6,28)=61.46, p<0.001$ ). These results demonstrate that repeated heat stress changed the regulation of HSP70 and GRP 78 expressions and caspase-3 activation in the $\mathrm{SN}$ of the mouse brain.

\section{DISCUSSION}

This study demonstrated that heat exposure impaired nigrostriatal dopaminergic neurons and motor function and increased dopamine turnover ratio. We also found that heat exposure changed of HSP70 expression and caspase-3 activity in the $\mathrm{SN}$ of mice brains.

First, to investigate whether repeated heat stress causes dysfunction of nigrostriatal dopaminergic neurons, we conducted behavioral, histological, and neurochemical analyses. Generally, the roles of striatal dopamine release and motor coordination are recognized; evidence from primates has shown that dopaminergic neurons are activated during movement, including dopamine release during motor sequence execution, dopamine depletion in PD patients, and the effectiveness of L-dopa therapy for PD. ${ }^{12,16)}$ We found that repeated heat stress reduced the dopaminergic cell bodies in the $\mathrm{SN}$ and 

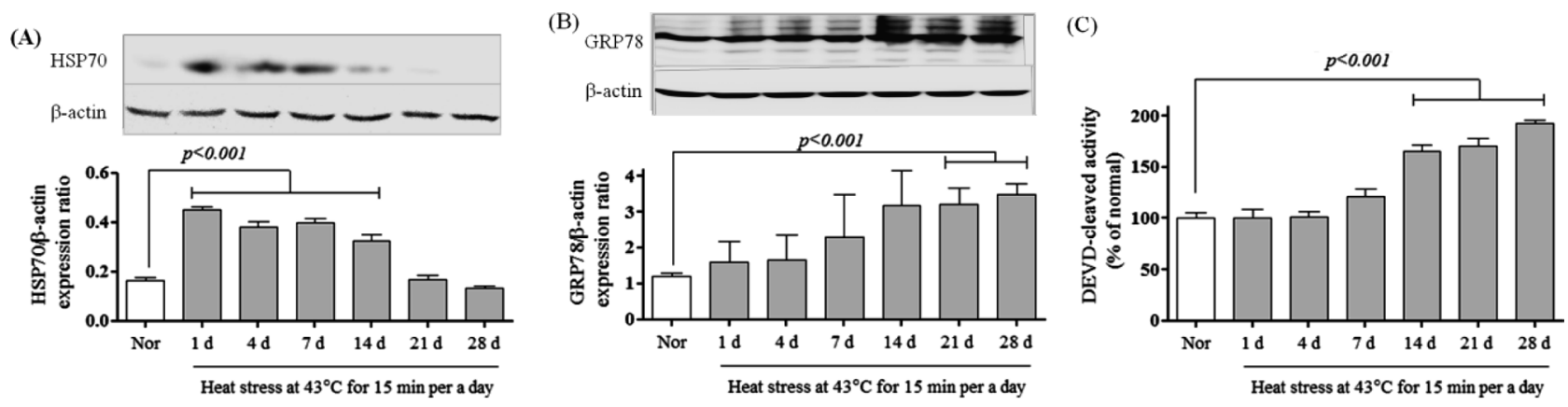

Fig. 4. Effect of Heat Stress on HSP70 and GRP78 Expressions and Caspase-3 Activity in the SN

After the mice were exposed to heat for different numbers of days, the SN was dissociated and lysed to measure the HSP70 expression (A), GRP78 expression (B), and caspase-3 activity (C). The graphs show the densitometric analysis of the ratio of expression of HSP70/ $\beta$-actin (A, $n=5)$ and the fluorescence of the cleaved substrate, $\operatorname{DEVD}(\mathrm{B}, n=5)$. Values are given as the mean \pm S.E.M. and compared with the normal group.

dopaminergic fibers in the striatum, resulting in bradykinesia and reduced movement distance (Figs. 1-3). Additionally, repeated heat stress increased the DOPAC, HVA, and DA turnover ratios and finally decreased dopamine level in the mouse striatum (Table 1). In the present study, dopamine depletion and motor dysfunction have occurred after different heat exposure days (28-d and 14-d, respectively). We assumed that this difference might be because movement controlling circuitry contains various neurotransmitters and neuromodulators from both dopaminergic neurons and non-dopaminergic neurons, presenting motor response complications. ${ }^{17,18)}$ Reports have suggested that acute heat stress upregulates dopamine in several brain regions ${ }^{3,19)}$ and the excess dopamine must be metabolized. ${ }^{20)}$ Dopamine forms DOPAC and HVA via the action of catechol- $O$-methyltransferase and monoamine oxidase. ${ }^{21)}$ This enzyme-catalyzed reaction, increases in the DOPAC/ DA and HVA/DA ratios (DA turnover ratio), affects dopamine oxidation, resulting in the generation a spectrum of toxic species, including hydrogen peroxide, oxygen radicals, semiquinones, and quinines, resulting in dopaminergic system activity dysfunction. ${ }^{11,20)}$ From the above reports, increased dopamine metabolism by repeated heat stress may partially contribute to the impairment of the nigrostriatal dopaminergic system and motor function in this study.

Next, to explore the mechanisms of repeated heat stress in the dysfunction of the nigrostriatal dopaminergic system, we measured HSP70 and GRP78 expressions and caspase-3 activity in mouse SN tissue. HSP70 is a member of the HSP family. These proteins are upregulated in neurons following a wide range of physiological and environmental stresses. ${ }^{1)}$ The functions of HSP70 are related to cellular thermotolerance and cytoprotection. ${ }^{8}$ For example, exposure to heat results in the constitutive elevation of cellular HSP70 to prevent protein damage and cellular destruction by interfering with apoptosis and restoring function to damaged proteins, thereby giving cells time to repair damage. ${ }^{22)}$ As in previous reports, we found that HSP70 expression was elevated in the SN of the mouse brain with heat exposure for up to $14 \mathrm{~d}$, providing cytoprotection upon thermal stress. By contrast, after heat exposure for $21 \mathrm{~d}$, HSP70 expression was decreased to the normal (Fig. 4A). During continuous exposure to heat, elevation of cellular HSP is reserved and HSP isoforms are redistributed. ${ }^{22)}$ That might be because of decreased binding of the heat shock factor 1 (HSF-1) to the heat shock element (HSE) or inadequate expression of HSF-1, reducing HSP syn- thesis in which has shown in degenerative neurons. ${ }^{223)}$ Thus, we assumed that the attenuated HSP70 expression after heat exposure for $21 \mathrm{~d}$ might be due to reduction of HSP synthesis with remaining studies. In addition, GRP 78 is a key mediator of the Unfolded Protein Response. Accumulation of unfolded proteins leads to dissociation of GRP78 from stress sensors for survival I early stage and apoptosis in late stage. ${ }^{15,24)}$ In the present study, we found that GRP78 expression gradually elevated in the $\mathrm{SN}$ of the mouse brain with heat exposure. Collectively, repeated heat exposure dysrugulated HSP70 and GRP78 expressions in the SN.

A number of studies have suggested that HSP70 inhibits neuronal apoptosis by modulating the mitochondrial proapoptotic or apoptotic proteins upstream and the apoptotic protease-activating factor 1 caspase-dependent and apoptosisinducing factor caspase-independent pathways downstream, ultimately resulting in the inhibition of neuronal cell death. ${ }^{25}$ ) In the present study, we found that caspase-3 activity was elevated with heat exposure for up to $14 \mathrm{~d}$. The data did not show exact correlation between the ability of HSP70, the inhibition of apoptosis, and the rescue of dopaminergic cells in the SN of the mouse brain with heat exposure. We thought that these contradictions might arise from complications of thermotolerance and neuronal death mechanisms. ${ }^{26,27)}$ Thus, additional research such as pharmacological inhibition of caspase- 3 or genetic knock-down will be necessary to establish the correlation between HSP70, caspase-3, and dopaminergic neuron dysfunction. However, we ascertained time-dependent effects of heat stress on HSP70 expression and caspase-3 activation in the $\mathrm{SN}$ of the mouse brain.

In conclusion, our data suggest that reduced nigrostriatal dopaminergic neurons after repeated heat exposure lead to decreased dopamine availability, which, in turn, may play an essential role in motor function impairment in mice. The findings clarified the relationships between repeated hyperthermia and changes in brain function and neurotransmitters, although further studies on mechanism and influence in other neuronal system are required.

Acknowledgment This research was supported by a grant from the Korea Institute of Oriental Medicine (KIOM) [K12274]. 


\section{REFERENCES}

1) Banerjee Mustafi S, Chakraborty PK, Dey RS, Raha S. Heat stress upregulates chaperone heat shock protein 70 and antioxidant manganese superoxide dismutase through reactive oxygen species (ROS), p38MAPK, and Akt. Cell Stress Chaperones, 14, 579-589 (2009).

2) Sinha RK. An approach to estimate EEG power spectrum as an index of heat stress using backpropagation artificial neural network. Med. Eng. Phys., 29, 120-124 (2007).

3) Harikai N, Tomogane K, Miyamoto M, Shimada K, Onodera S, Tashiro S. Dynamic responses to acute heat stress between 34 degrees $\mathrm{C}$ and 38.5 degrees $\mathrm{C}$, and characteristics of heat stress response in mice. Biol. Pharm. Bull., 26, 701-708 (2003).

4) Xia Z, Zheng X, Zheng H, Liu X, Yang Z, Wang X. Cold-inducible RNA-binding protein (CIRP) regulates target mRNA stabilization in the mouse testis. FEBS Lett., 586, 3299-3308 (2012).

5) Garcia C, Schmitt P, D'Aléo P, Bittel J, Curé M, Pujol JF. Regional specificity of the long-term variation of tyrosine hydroxylase protein in rat catecholaminergic cell groups after chronic heat exposure. J. Neurochem., 62, 1172-1181 (1994).

6) Khan VR, Brown IR. The effect of hyperthermia on the induction of cell death in brain, testis, and thymus of the adult and developing rat. Cell Stress Chaperones, 7, 73-90 (2002).

7) White MG, Emery M, Nonner D, Barrett JN. Caspase activation contributes to delayed death of heat-stressed striatal neurons. $J$. Neurochem., 87, 958-968 (2003).

8) Xiao C, Mileva-Seitz V, Seroude L, Robertson RM. Targeting HSP70 to motoneurons protects locomotor activity from hyperthermia in Drosophila. Dev. Neurobiol., 67, 438-455 (2007).

9) Yang YL, Lin MT. Heat shock protein expression protects against cerebral ischemia and monoamine overload in rat heatstroke. Am. J. Physiol., 276, H1961-H1967 (1999).

10) White MG, Saleh O, Nonner D, Barrett EF, Moraes CT, Barrett JN. Mitochondrial dysfunction induced by heat stress in cultured rat CNS neurons. J. Neurophysiol., 108, 2203-2214 (2012).

11) Chinta SJ, Andersen JK. Dopaminergic neurons. Int. J. Biochem. Cell Biol., 37, 942-946 (2005).

12) Marsden CA. Dopamine: the rewarding years. Br. J. Pharmacol., 147 (Suppl. 1), S136-S144 (2006).

13) Isomura Y, Takekawa T, Harukuni R, Handa T, Aizawa H, Takada M, Fukai T. Reward-modulated motor information in identified striatum neurons. J. Neurosci., 33, 10209-10220 (2013).

14) Yang TH, Ho WY, Shih MF, Leu KL, Wen YS, Liu CC. Effects of combination treatment with dexamethasone and mannitol on neuronal damage and survival in experimental heat stroke. Biol. Pharm.
Bull., 33, 1522-1528 (2010).

15) Lee AS. The ER chaperone and signaling regulator GRP78/BiP as a monitor of endoplasmic reticulum stress. Methods, 35, 373-381 (2005).

16) Lappin JM, Reeves SJ, Mehta MA, Egerton A, Coulson M, Grasby PM. Dopamine release in the human striatum: motor and cognitive tasks revisited. J. Cereb. Blood Flow Metab., 29, 554-564 (2008).

17) Fox SH, Brotchie JM, Lang AE. Non-dopaminergic treatments in development for Parkinson's disease. Lancet Neurol., 7, 927-938 (2008).

18) Metman LV, Konitsiotis S, Chase TN. Pathophysiology of motor response complications in Parkinson's disease: hypotheses on the why, where, and what. Mov. Disord., 15, 3-8 (2000).

19) Yeghiayan SK, Georgelis JH, Maher TJ, Lieberman HR. Beneficial effects of a protein free, high carbohydrate meal on rat coping behavior and neurotransmitter levels during heat stress. Nutr. Neurosci., 7, 335-340 (2004).

20) Satoh K, Nonaka R, Ohashi N, Shimizu M, Oshio S, Takeda K. The effects of in utero exposure to a migrant, 4,4'-butylidenebis(6-tbutyl- $m$-cresol), from nitrile-butadiene rubber gloves on monoamine neurotransmitter in rats. Biol. Pharm. Bull., 31, 2211-2215 (2008).

21) Mauriño R, Machado A, Santiago M. Effect of in vivo striatal perfusion of lipopolysaccharide on dopamine metabolites. Neurosci. Lett., 475, 121-123 (2010).

22) Horowitz M, Robinson SD. Heat shock proteins and the heat shock response during hyperthermia and its modulation by altered physiological conditions. Prog. Brain Res., 162, 433-446 (2007).

23) Taylor DM, Tradewell ML, Minotti S, Durham HD. Characterizing the role of Hsp90 in production of heat shock proteins in motor neurons reveals a suppressive effect of wild-type Hsf1. Cell Stress Chaperones, 12, 151-162 (2007).

24) Gorbatyuk MS, Gorbatyuk OS. The molecular chaperone GRP78/ $\mathrm{BiP}$ as a therapeutic target for neurodegenerative disorders: a mini review. J. Genet. Syndr. Gene Ther., 4, 128 (2013).

25) Sabirzhanov B, Stoica BA, Hanscom M, Piao CS, Faden AI. Overexpression of HSP70 attenuates caspase-dependent and caspase-independent pathways and inhibits neuronal apoptosis. J. Neurochem., 123, 542-554 (2012).

26) Basiricò L, Morera P, Primi V, Lacetera N, Nardone A, Bernabucci U. Cellular thermotolerance is associated with heat shock protein 70.1 genetic polymorphisms in Holstein lactating cows. Cell Stress Chaperones, 16, 441-448 (2011).

27) White MG, Luca LE, Nonner D, Saleh O, Hu B, Barrett EF, Barrett JN. Cellular mechanisms of neuronal damage from hyperthermia. Prog. Brain Res., 162, 347-371 (2007). 